\title{
Real-time in vivo computed optical interferometric tomography
}

\author{
Adeel Ahmad ${ }^{1,2}$, Nathan D. Shemonski, ${ }^{1,2}$, Steven G. Adie ${ }^{1,2}$, Hee-Seok Kim², Wen-Mei W. Hwu², \\ P. Scott Carney ${ }^{1,2}$ and Stephen A. Boppart ${ }^{1,2,3,4 \star}$
}

\begin{abstract}
High-resolution real-time tomography of scattering tissues is important for many areas of medicine and biology ${ }^{1-6}$. However, the compromise between transverse resolution and depth-of-field, in addition to low sensitivity deep in tissue, continues to impede progress towards cellular-level volumetric tomography. Computed imaging has the potential to solve these long-standing limitations. Interferometric synthetic aperture microscopy ${ }^{7-9}$ is a computed imaging technique enabling high-resolution volumetric tomography with spatially invariant resolution. However, its potential for clinical diagnostics remains largely untapped because full volume reconstructions required lengthy post-processing, and the phase-stability requirements have been difficult to satisfy in vivo. Here, we demonstrate how three-dimensional Fourier-domain resampling, in combination with high-speed optical coherence tomography, can achieve high-resolution in vivo tomography. Enhanced depth sensitivity was achieved over a depth of field extended in real time by more than an order of magnitude. This work lays the foundation for high-speed volumetric cellular-level tomography.
\end{abstract}

A number of approaches to achieve optimal resolution over large depth ranges in optical coherence tomography (OCT) have been reported previously; these include hardware solutions, software additions, or a combination of the two. Such solutions include focus shifting ${ }^{10,11}$, as used in confocal microscopy ${ }^{12}$, imaging with multiple beams, each focused at different depths within the sample ${ }^{13}$, illuminating the sample with a Bessel beam ${ }^{14,15}$, and numerical refocusing or deconvolution methods ${ }^{16,17}$. Although producing high-quality tomograms, these methods suffer from long acquisition or processing times, or they require complex optical set-ups for which the design or manufacture of compact systems becomes difficult. A common difficulty with computed imaging techniques, often preventing them from widespread adoption, is their large computational requirements. Digital holographic microscopy $(\mathrm{DHM})^{18}$, interferometric synthetic aperture microscopy (ISAM), computational adaptive optics $(\mathrm{CAO})^{19}$ and holoscopy ${ }^{20}$ all require the manipulation of acquired two-dimensional (2D) or three-dimensional (3D) data in the Fourier domain, typically resulting in lengthy processing times. This disparity between imaging and processing rates hinders the usefulness of any given modality. Without realtime implementations to provide feedback to the user during imaging, high-quality images can be elusive, requiring several imaging sessions or extensive post-processing.

In some modalities such as magnetic resonance imaging (MRI), ISAM and holoscopy, computing needs can be greatly reduced by performing a single coordinate warping (resampling) in the Fourier domain in lieu of more expensive reconstruction methods ${ }^{21}$. Even so, because of the computational requirements of the 3D Fourier-domain resampling, real-time implementations for modalities such as ISAM and holoscopy have remained out of reach. Here we report a new method for performing 3D Fourierdomain resampling by decomposing the $3 \mathrm{D}$ problem into two sets of $2 \mathrm{D}$ subproblems, which enables real-time computed imaging on high-speed but memory-limited devices such as graphics processing units (GPUs). With this advance in Fourier-domain resampling, our software is able to perform ISAM in the steady state and maintain an input-output flow at the camera-limited $92 \mathrm{kHz}$ A-scan rate (95 frames per second). Real-time ISAM feedback enables the development of a system for scanning and mounting in vivo samples in a phase-stable way that minimizes or eliminates phase noise artefacts. To this end, we demonstrate high-resolution in vivo computed tomography over an extended depth, which can now potentially enable practical applications such as intraoperative and functional imaging.

Mathematically, ISAM is a solution to the inverse scattering problem that relates the Fourier transform of the measured data to the Fourier transform of the scattering potential of the object to be reconstructed. In general, ISAM is implemented as a Fourier domain resampling of the form $k=f\left(q_{z}, \mathbf{q}\right)$, where $\mathbf{q}=\left(q_{x}, q_{y}\right)$ and $q_{z}$ are the transverse and axial spatial frequency coordinates, respectively. The added processing and the prohibitively large memory requirements for storing the $3 \mathrm{D}$ complex volumetric data before Fourier-domain resampling makes ISAM and other computed imaging techniques computationally more expensive than standard approaches. In this Letter, we report a method for performing Fourier-domain resampling by decomposing a $3 \mathrm{D}$ problem into two sets of $2 \mathrm{D}$ subproblems. This is achieved by writing the required coordinate warping as the composition of two functions, each of which only depends on one transverse spatial frequency, $k=g_{1}\left(g_{2}\left(q_{z}, q_{y}\right), q_{x}\right)$. Specifically for ISAM, we allow $g_{1}\left(q_{z}, q\right)=1 / 2 \sqrt{ }\left(\left(2 q_{z}\right)^{2}+q^{2}\right)$ and $g_{2}\left(q_{z}, q\right)=1 / 2 \sqrt{q_{z}^{2}+q^{2}}$. Utilizing $g_{1}$ and subsequently $g_{2}$, exact $3 \mathrm{D}$ ISAM is possible without requiring a complete $3 \mathrm{D}$ Fourier-domain representation of the tomogram. This enables steady-state, memory-efficient, frameby-frame data transfer and computation, which is the basis of our real-time implementation. A detailed description of the resampling decomposition is included in Supplementary Section S1.

A 1,330 nm spectral-domain optical coherence tomography (SDOCT) system was used to demonstrate the real-time imaging capabilities of in vivo 3D ISAM in highly scattering biological tissues at

'Beckman Institute for Advanced Science and Technology, University of Illinois at Urbana-Champaign, 405 N. Mathews Ave, Urbana, Illinois 61801, USA, ${ }^{2}$ Department of Electrical and Computer Engineering, University of Illinois at Urbana-Champaign, 1406 W. Green St, Urbana, Illinois 61801, USA, ${ }^{3}$ Department of Bioengineering, University of Illinois at Urbana-Champaign, 1304 W. Springfield Avenue, Urbana, Illinois 61801, USA, ${ }^{4}$ Department of Internal Medicine, University of Illinois at Urbana-Champaign, 506 S. Mathews Ave, Urbana, Illinois 61801, USA; 'These authors contributed equally to this work. *e-mail: boppart@illinois.edu 
a

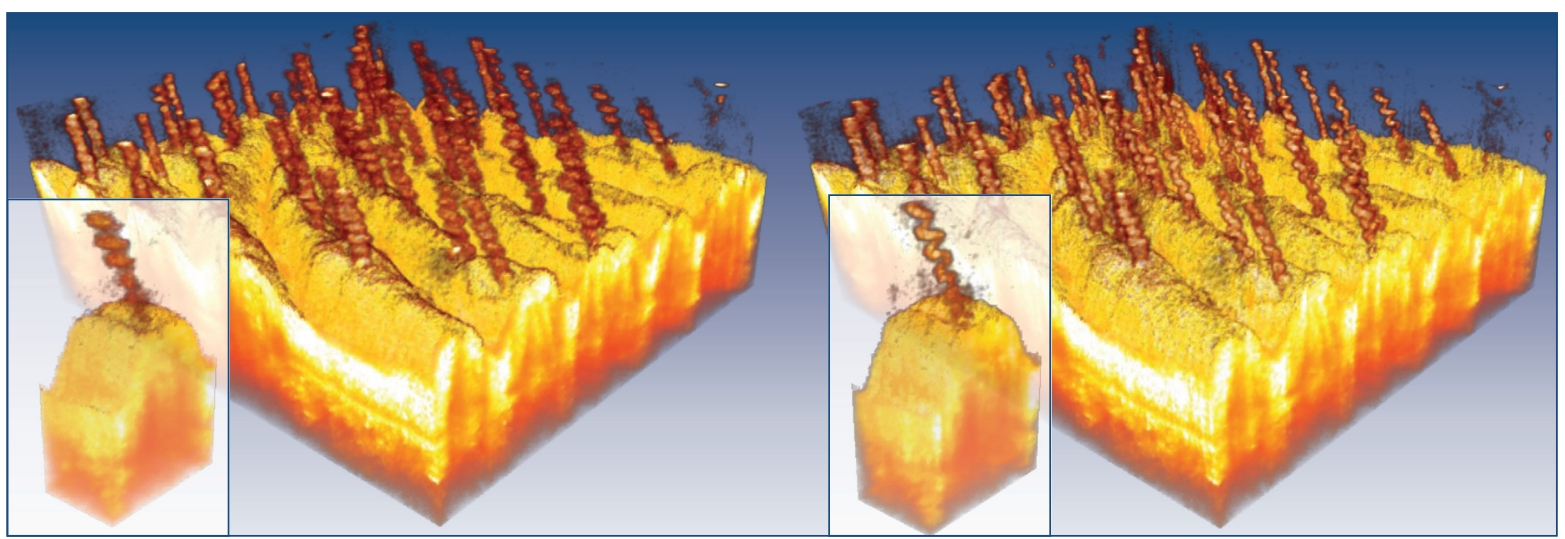

b

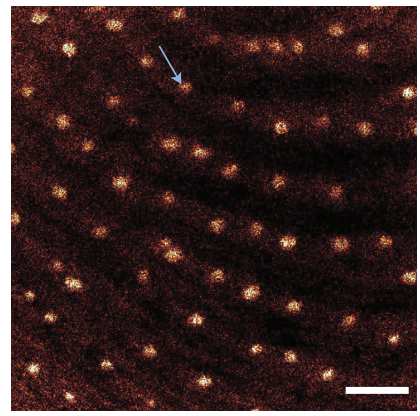

c

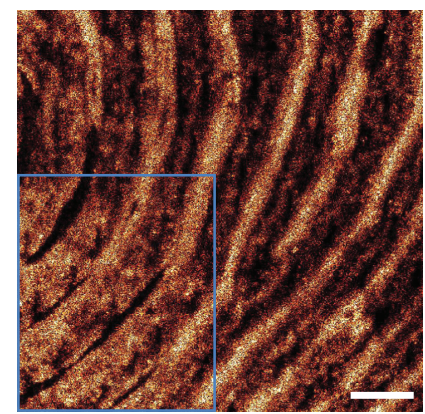

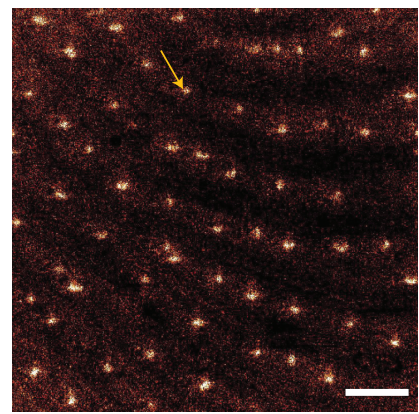
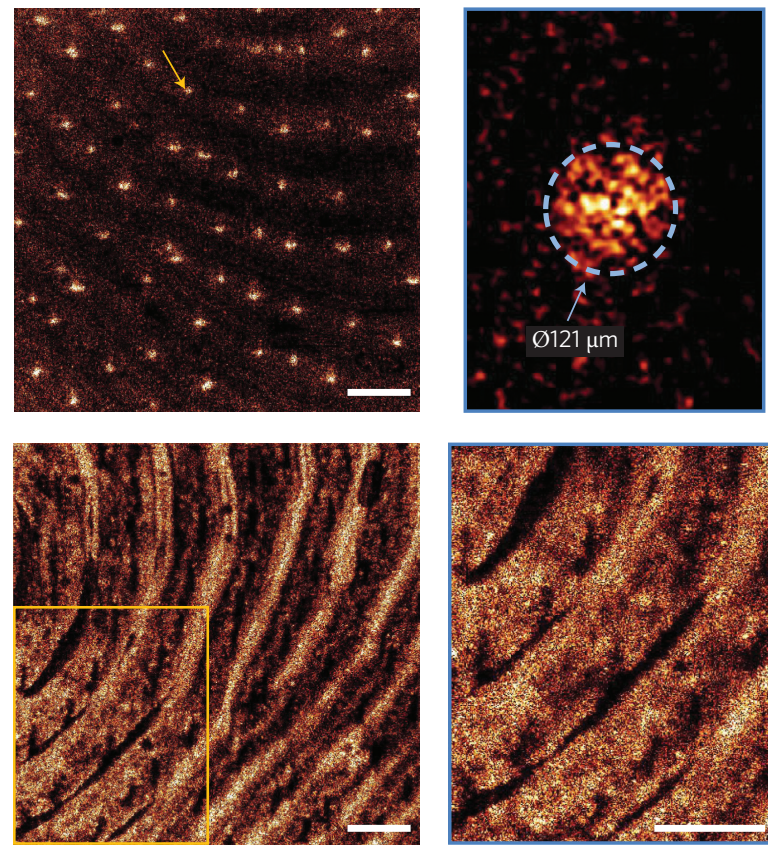

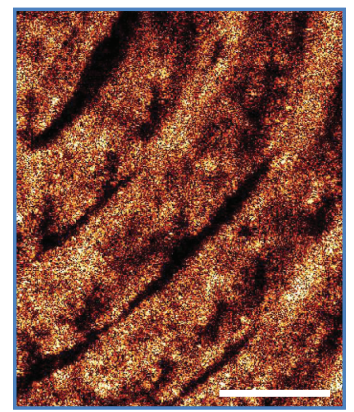

Figure 1 | Real-time in vivo ISAM of healthy human skin from the fingerprint region of the index finger. a, Three-dimensional rendering comparing OCT (left) and ISAM (right). The spiral structures of the sweat ducts appear with higher resolution and higher SNR in the ISAM data set (Supplementary Movie S2). b, Representative en face planes (OCT left and ISAM right) with enlarged representative regions indicated by colour-coded arrows (blue, OCT; yellow, ISAM) showing the cross-section of the sweat ducts. The diameter of the sweat ducts obtained with ISAM more closely matches the known anatomical range of diameters. c, En face planes (OCT left and ISAM right) at a (optical) depth of $780 \mu \mathrm{m}$ below the surface, showing enhanced resolution deeper inside the superficial dermis. Scale bars, $500 \mu \mathrm{m}$.

the full acquisition rate of the SD-OCT system (Supplementary Fig. S1). Three-dimensional ISAM was implemented on a single GPU, with highly efficient memory utilization (Supplementary Fig. S2). To this end, our Fourier-domain resampling method combined with the parallel processing capabilities of the GPU were exploited to achieve several orders of magnitude improvement in processing speed compared to previous CPU implementations ${ }^{22}$, limited only by the line rate of our camera.

After validating our method on a tissue-mimicking phantom (Supplementary Fig. S3 and Movie S1), we demonstrated our realtime capabilities for obtaining in vivo ISAM tomograms. Reliable acquisition of phase-sensitive measurements in vivo is challenging because of the sample-induced motions in living tissue and inherent system noise. As ISAM relies on precise phase relationships throughout an acquired tomogram, its reconstruction quality is also susceptible to these sources of phase noise. To avoid any lengthy or complex motion correction procedures, multiple steps were taken to acquire phase-stable data. Our high-speed data acquisition helped in minimizing motion artefacts and phase noise. In addition, custom galvanometer waveforms for lateral beam scanning, as well as proper tissue mounting, combined to enable in vivo ISAM. The capability to visualize $3 \mathrm{D}$ ISAM data in real time enabled us to ensure proper imaging parameters for optimum ISAM reconstructions and to minimize the impact of axial and transverse movement, thus ensuring repeatable and reliable measurements. Figure 1 and Supplementary Movie S2 demonstrate in vivo ISAM on the skin of a healthy human volunteer spanning a transverse field of view of $3.2 \mathrm{~mm} \times 3.2 \mathrm{~mm}$. The tomogram reveals the spiral structure of the sweat ducts characteristic of thick skin and, qualitatively, it is clear that neither motion nor phase noise corrupted the ISAM reconstruction. To verify this quantitatively, a random selection of sweat ducts was chosen and the crosssectional diameters were measured. For standard OCT, the mean diameter was $114 \mu \mathrm{m}(\sigma=11 \mu \mathrm{m})$ and for ISAM the mean diameter was $61.5 \mu \mathrm{m}(\sigma=8.0 \mu \mathrm{m})$. The actual cross-sectional diameter of sweat ducts is known to range from $50 \mu \mathrm{m}$ to $80 \mu \mathrm{m}$ (ref. 23), verifying that ISAM provides anatomically accurate reconstruction of tissue structure in vivo. 

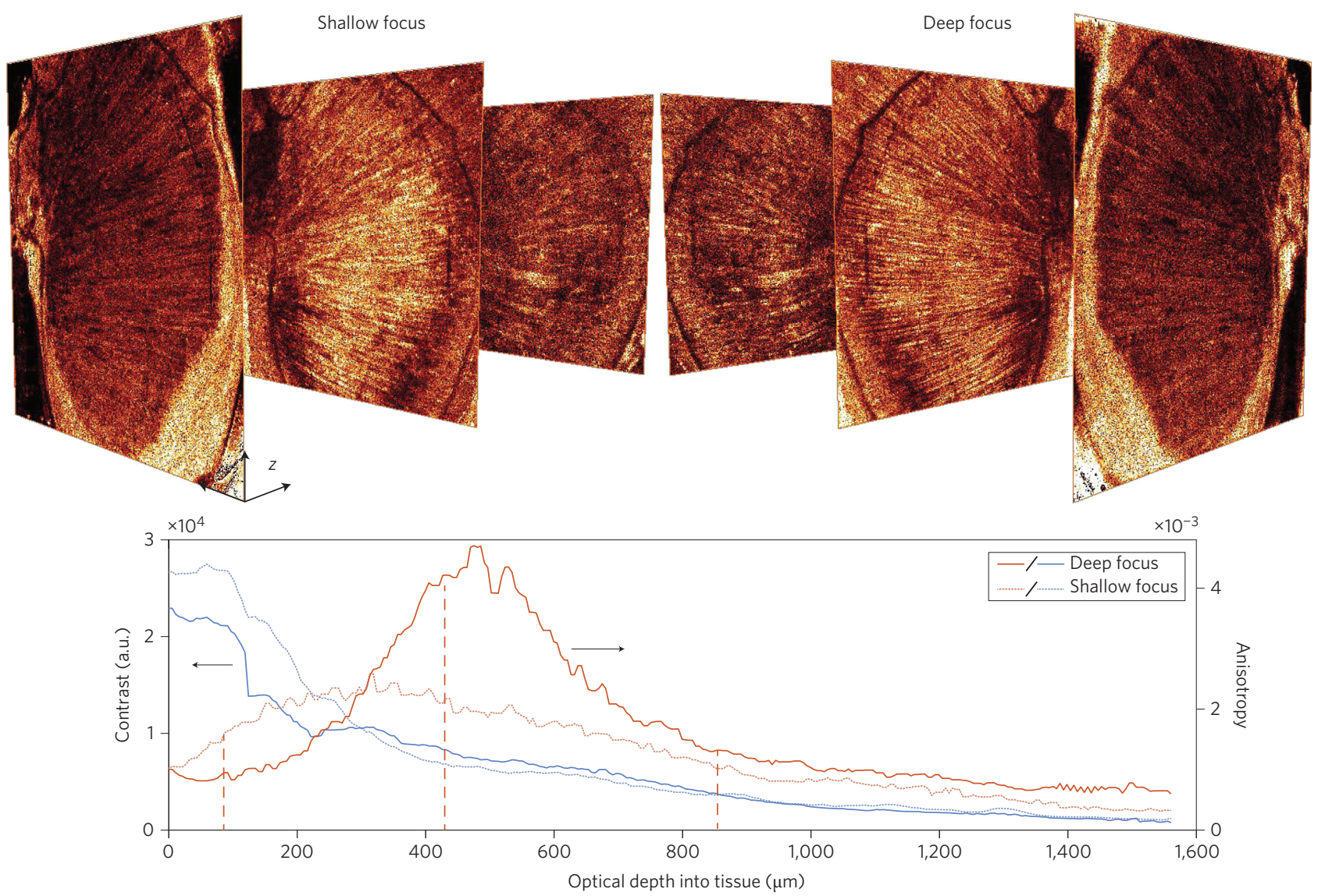

Figure $\mathbf{2}$ | Focus placement with real-time ISAM on ex vivo mouse muscle. Left image stack: three en face planes (depths denoted as dashed lines in the chart) from an ISAM tomogram with a focus placed shallow $(310 \mu \mathrm{m})$ in the tissue. Right image stack: corresponding slices from an ISAM tomogram with the focus placed deeper $(1,000 \mu \mathrm{m})$ in the sample. The tomogram with a deep focus has a slight loss in signal near the surface, but deep in the sample the strategically placed focus enhances fine muscle structures. These results are quantified using anisotropy and contrast as image quality metrics in the chart. The en face planes shown have transverse dimensions of $3.2 \mathrm{~mm} \times 3.2 \mathrm{~mm}$.

We then investigated the influence of focus placement on depth-dependent image quality using ex vivo mouse muscle. When conducting tomography with a high-numerical-aperture (NA) optical beam, we found that real-time, tissue-dependent focus placement was crucial, and strongly affected the depthdependent sensitivity of the resulting tomogram. Figure 2 compares two ISAM tomograms, one with the focus placed near the surface and the other with a deeply placed focus. We see that with the deeper focus, there is practically no loss of information near the tissue surface. Deep in the tissue, however, where (due to the effects of optical scattering) there is naturally very low sensitivity, the image quality is dramatically improved with the deeper focus. We attribute this to the enhanced collection of singly backscattered light when the focus is placed deep in the sample. Real-time feedback is thus beneficial for optimizing depth-dependent image quality for a given tissue type. Image quality metrics, such as the anisotropy ${ }^{24}$ and contrast shown in Fig. 2, validate our results for deep focus placement.

We finally combine in vivo imaging with a strategically placed focus to image $1.2 \mathrm{~mm}$ (optical depth) in highly scattering healthy human skin on the wrist. Without sacrificing transverse resolution far from focus, we extend the depth-of-field by over an order of magnitude (24 Rayleigh ranges) in real time. Figure 3 demonstrates that, away from focus, not only does ISAM reveal the true structure obscured in OCT, but it also recovers proper constructive interference, leading to an increase in signal-to-noise ratio (SNR). With our simplistic optical set-up, we can achieve a resolution, penetration depth, and sensitivity comparable to those of more complex imaging systems $s^{25}$. Clearly resolved in Fig. $3 \mathrm{~b}$ are the stratum disjunction (SD), stratum corneum (SC), reticular dermis (RD) and subcutaneous fat (SF). In Fig. 3a,c, the RD (a skin layer containing a network of small blood vessels) suffers from a large amount of blurring due to defocus. Figure $3 \mathrm{~b}$,d shows the resulting ISAM reconstruction where these features are brought back into focus. This shows that ISAM produces better quality volumes over large depth ranges by improving two important properties used to measure the quality of images: resolution and SNR.

In conclusion, the decomposition of Fourier-domain resampling with high-speed data acquisition coupled with real-time feedback has enabled the first demonstration of in vivo 3D ISAM. Our results, both qualitatively and quantitatively, show the ability of ISAM and strategic focus placement to enhance both the resolution and sensitivity throughout an extended volume. Furthermore, by combining this technique with $\mathrm{CAO}^{19}$, computed optical imaging may have the capability to provide aberration-free 3D tomography that can complement or replace more complicated optical set-ups for high-resolution imaging. By using a higher-NA optical set-up in conjunction with these computational techniques, future work will pursue real-time cellular resolution tomography over an extended depth range. These advances for broadband computed interferometric tomography provide the capability of high-resolution deep-tissue volumetric imaging, with applications in time-sensitive situations such as image-guided procedures or optical biopsy for clinical screening applications. 

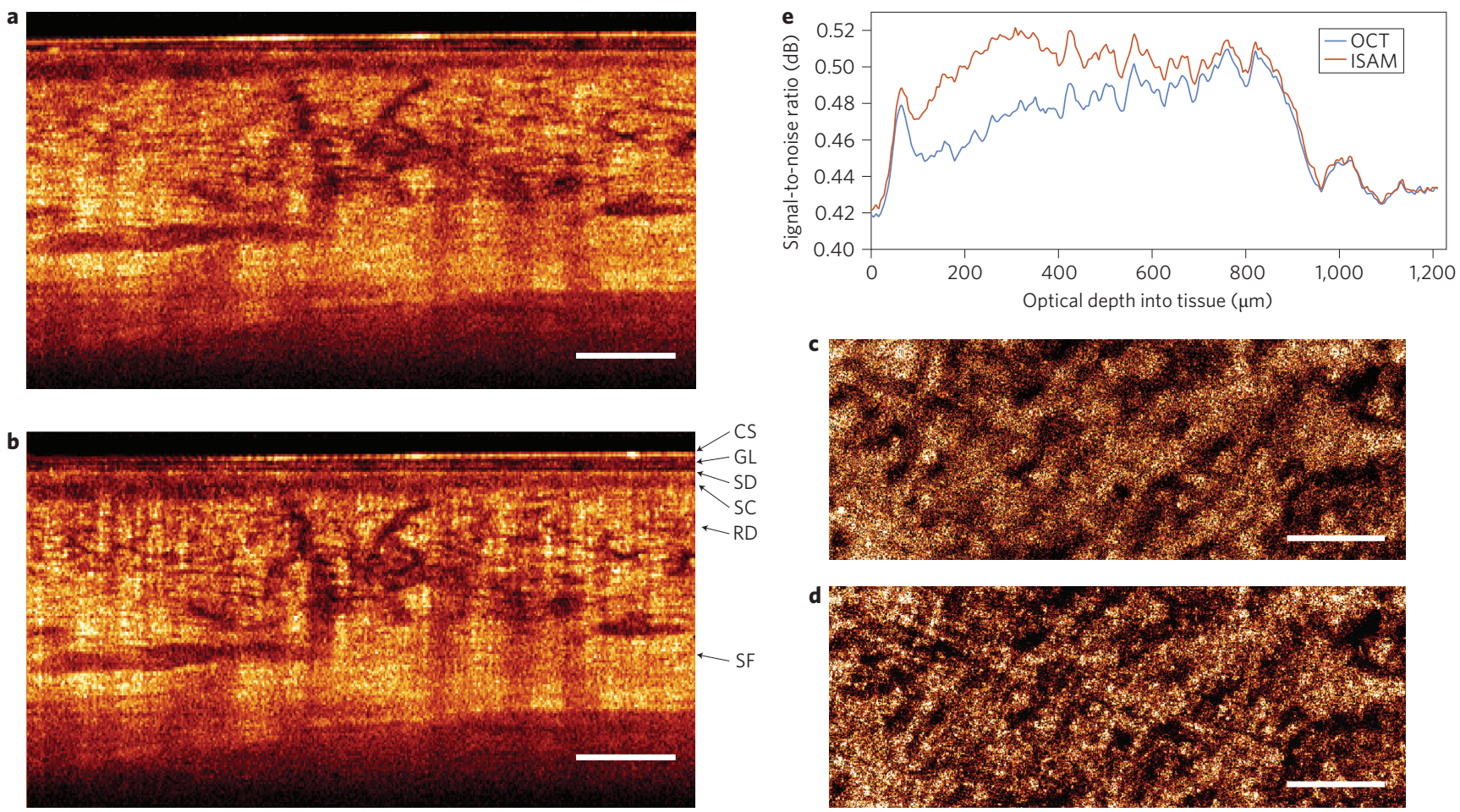

Figure 3 | Real-time in vivo ISAM of skin from a healthy human wrist. The optical focus was placed $1.2 \mathrm{~mm}$ deep inside the tissue, and the depth of field was extended by over an order of magnitude (24 Rayleigh ranges) in real time. a,b, Representative cross-sectional plane of OCT (a) and ISAM (b) processed data set. CS, coverslip; GL, glycerol; SD, stratum disjunction; SC, stratum corneum; RD, reticular dermis; SF, subcutaneous fat. c,d, Cropped en face planes of OCT (c) and ISAM (d) at an optical depth of $520 \mu \mathrm{m}$ into the tissue. e, Variation of SNR with depth. Compared to OCT, ISAM shows significant improvement over an extended depth range. Scale bars, $500 \mu \mathrm{m}$.

\section{Methods}

Experimental set-up. Imaging was performed using the $1,330 \mathrm{~nm}$ SD-OCT system shown in Supplementary Fig. S1. A superluminescent diode (SLD) with a bandwidth of $105 \mathrm{~nm}$ was used as the light source, giving an axial resolution of $7.4 \mu \mathrm{m}$ (full width at half maximum) in air. An identical pair of achromatic doublets with focal lengths of $30 \mathrm{~mm}$ were used to image the fibre core to the sample, resulting in $\mathrm{NA}=0.1$. The Rayleigh range was calculated to be $50 \mu \mathrm{m}$ in air (using $4.6 \mu \mathrm{m}$ as the radius of the beam waist $\left(1 / \mathrm{e}^{2}\right.$ half-width)), and the optical imaging depth (physical depth $\times$ refractive index) from the spectrometer (BaySpec) was $2.8 \mathrm{~mm}$. The beam was raster-scanned along the fast-scanning and slow-scanning axes over the sample. Customized driving waveforms ( $85 \%$ linear, $15 \%$ flyback) for the $x-y$ galvanometer pair (SCANLAB, SCANcube 7) were generated by a DAQ board (National Instruments, NI-PCIe-6353), which also generated a control signal to synchronize the scanning with the acquisition from the InGaAs line-scan camera (Goodrich, SU-LDH2). The camera was operated at the maximum A-scan rate of $91.912 \mathrm{kHz}$ and was interfaced through an image acquisition board (National Instruments, NI-PCIe-1427). The software for data acquisition and graphical user interface was developed in LabVIEW and the data were processed in real time through dynamic link library (DLL) function calls implemented in C (Microsoft Visual Studio 2008 environment). A computer with an Intel Core i7 processor (operating at $3.3 \mathrm{GHz}, 12 \mathrm{~GB}$ DDR3 RAM) was used for running the system, and the compute unified device architecture (CUDA) extension v4.1 from NVIDIA was used for GPU kernel calls on the NVIDIA GeForce GTX 580 GPU. The ex vivo samples were placed on a three-axis stage separate from the beam collimator and scanning galvanometers, and the in vivo data were taken by gently pressing the tissue of interest onto a custom mount attached to the SCANcube and beam collimator. By attaching a coverslip mount directly to the lens tube containing the objective lens, which was attached to the scanning optics and fibre collimator, the phase stability requirements for ISAM could be met reliably. Acquired volumes consisted of 1,024 pixels per A-scan and $810 \mathrm{~A}$-scans along each transverse dimension, resulting in an acquisition time of $\sim 8.5 \mathrm{~s}$.

GPU implementation. A multistreamed GPU program was developed for real-time 3D ISAM (Supplementary Fig. S2) based on the resampling decomposition. The streams executed concurrently, performing two orthogonal 2D ISAM reconstructions where one stream processed the fast-axis frames and the other stream processed the orthogonal dimension of the previously acquired 2D ISAM processed data. This decomposition required only a single volume buffer in GPU memory at any given time because each previously acquired slow-axis frame can be replaced by the currently acquired fast-axis frame. All the processing on the GPU was done with single precision floating point operations, and gamma correction was applied on the processed data for display purposes. In this Letter, the term 'real-time' means that data acquisition was never paused for processing, and that data are continuously being updated for the user, although the data being displayed are at a small latency of one volume ( $8.5 \mathrm{~s}$ for the dimensions mentioned in the previous section). If we were to allow the camera to pause between volume acquisitions to complete ISAM reconstruction, the latency would be reduced even further (to $1.5 \mathrm{~s}$ for the same volume). See Supplementary Section S2 for further details.

Image display and analysis. To remove any bias in displaying comparisons between OCT and ISAM, all comparisons were viewed on the same histogram scale using the same upper and lower saturation values. In real time, our system has the ability to both process and display cross-sectional and user-selected en face planes in the $2 \mathrm{D}$ and $3 \mathrm{D}$ ISAM reconstructions. In post-processing for further visualization, the calculated energy in each ISAM tomogram (en face plane by en face plane) was normalized to that of the OCT tomogram. After power-normalization, the noise floors in the OCT and ISAM tomograms were in excellent agreement. To further visualize complete OCT and ISAM data sets, the tomograms were depth-normalized to a common histogram scale. The 3D volume rendering in Fig. 1 was performed in Amira 5.4.2. The volume was first segmented into two regions, the stratum corneum/stratum lucidum (containing the sweat ducts) and the dermis, then different colour maps were used for each. Image metrics such as anisotropy, SNR, and contrast were computed to quantitatively evaluate the improvement in reconstruction quality. The anisotropy image quality metric was originally devised ${ }^{24}$ to be sensitive to additive Gaussian noise in incoherent imaging systems. Further testing on various tissue phantoms and tissue data (data not shown) confirmed that this metric is also reliable for noise present in OCT data. SNR and contrast were computed using the $20 \%$ (noise) and $90 \%$ (signal) quantiles of the intensity histograms.

Received 2 November 2012; accepted 4 March 2013; published online 21 April 2013

\section{References}

1. Boppart, S. A. et al. In vivo cellular optical coherence tomography imaging. Nature Med. 4, 861-865 (1998).

2. Adler, D. C. et al. Three-dimensional endomicroscopy using optical coherence tomography. Nature Photon. 1, 709-716 (2007). 
3. Liu, L. et al. Imaging the subcellular structure of human coronary atherosclerosis using micro-optical coherence tomography. Nature Med. 17, 1010-1014 (2011).

4. Vakoc, B. J. et al. Three-dimensional microscopy of the tumor microenvironment in vivo using optical frequency domain imaging. Nature Med. 15, 1219-1223 (2009).

5. Yun, S. H. et al. Comprehensive volumetric optical microscopy in vivo. Nature Med. 12, 1429-1433 (2006).

6. Drexler, W. et al. Ultrahigh-resolution ophthalmic optical coherence tomography. Nature Med. 7, 502-507 (2001).

7. Ralston, T. S., Marks, D. L., Carney, P. S. \& Boppart, S. A. Interferometric synthetic aperture microscopy. Nature Phys. 3, 129-134 (2007)

8. Davis, B. J., Marks, D. L., Ralston, T. S., Carney, P. S. \& Boppart, S. A. Interferometric synthetic aperture microscopy: computed imaging for scanned coherent microscopy. Sensors 8, 3903-3931 (2008).

9. Davis, B. J. et al. Nonparaxial vector-field modeling of optical coherence tomography and interferometric synthetic aperture microscopy. J. Opt. Soc. Am. A 24, 2527-2542 (2007).

10. Srinivasan, V. J., Radhakrishnan, H., Jiang, J. Y., Barry, S. \& Cable, A. E. Optical coherence microscopy for deep tissue imaging of the cerebral cortex with intrinsic contrast. Opt. Express 20, 2220-2239 (2012).

11. Rolland, J. P., Meemon, P., Murali, S., Thompson, K. P. \& Lee, K-S. Gaborbased fusion technique for optical coherence microscopy. Opt. Express 18, 3632-3642 (2010).

12. Rajadhyaksha, M., Gonzalez, S., Zavislan, J. M., Anderson, R. R. \& Webb, R. H. In vivo confocal scanning laser microscopy of human skin II: advances in instrumentation and comparison with histology. J. Invest. Dermatol. 113, 293-303 (1999).

13. Beau, A. S. et al. In vivo endoscopic multi-beam optical coherence tomography. Phys. Med. Biol. 55, 615-622 (2010).

14. Leitgeb, R. A., Villiger, M., Bachmann, A. H., Steinmann, L. \& Lasser, T. Extended focus depth for Fourier domain optical coherence microscopy. Opt. Lett. 31, 2450-2452 (2006)

15. Blatter, C. et al. Extended focus high-speed swept source OCT with selfreconstructive illumination. Opt. Express 19, 12141-12155 (2011)

16. Yasuno, Y. et al. Non-iterative numerical method for laterally superresolving Fourier domain optical coherence tomography. Opt. Express 14, 1006-1020 (2006).

17. $\mathrm{Yu}, \mathrm{L}$. et al. Improved lateral resolution in optical coherence tomography by digital focusing using two-dimensional numerical diffraction method. Opt. Express 15, 7634-7641 (2007).

18. Colomb, T. et al. Numerical parametric lens for shifting, magnification, and complete aberration compensation in digital holographic microscopy. J. Opt Soc. Am. A 23, 3177-3190 (2006).

19. Adie, S. G., Graf, B. W., Ahmad, A., Carney, P. S. \& Boppart, S. A. Computationa adaptive optics for broadband optical interferometric tomography of biological tissue. Proc. Natl Acad. Sci. USA 109, 7175-7180 (2012).
20. Hillmann, D., Franke, G., Lührs, C., Koch, P. \& Hüttmann, G. Efficient holoscopy image reconstruction. Opt. Express 20, 21247-21263 (2012).

21. Kim, M-K. Tomographic three-dimensional imaging of a biological specimen using wavelength-scanning digital interference holography. Opt. Express 7, 305-310 (2000).

22. Ralston, T. S., Marks, D. L., Carney, P. S. \& Boppart, S. A. Real-time interferometric synthetic aperture microscopy. Opt. Express 16, 2555-2569 (2008).

23. Wilke, K., Martin, A., Terstegen, L. \& Biel, S. S. A short history of sweat gland biology. Int. J. Cosmetic Sci. 29, 169-179 (2007).

24. Gabarda, S. \& Cristóbal, G. Blind image quality assessment through anisotropy. J. Opt. Soc. Am. A 24, B42-B51 (2007).

25. Blatter, C. et al. In situ structural and microangiographic assessment of human skin lesions with high-speed OCT. Biomed. Opt. Express 3, 2636-2646 (2012).

\section{Acknowledgements}

The authors thank E.J. Chaney (University of Illinois at Urbana-Champaign) for providing the tissue samples used throughout this study, D.L. Marks (formerly at University of Illinois at Urbana-Champaign) for providing the tissue phantom and D. Spillman (University of Illinois at Urbana-Champaign) for administrative and information technology support related to this research. This research was supported in part by grants from the National Institutes of Health (NIH; R01 EB012479 to S.A.B.) and an NIH Bioengineering Research Partnership (R01 EB013723 to S.A.B.). A.A. was funded in part by the NIH National Cancer Institute Alliance for Nanotechnology in Cancer (Midwest Cancer Nanotechnology Training Center; grant R25-CA154015A) and Texas Instruments.

\section{Author contributions}

Experimental data were acquired by A.A., N.D.S. and S.G.A. Analysis and interpretation of data was carried out by A.A., N.D.S. and S.G.A. Additional processing and visualization was performed by A.A. and N.D.S. GPU code was written by A.A., N.D.S. and H.K., and reviewed by W.W.H. The manuscript was written and edited by all authors. S.A.B. and P.S.C. made seminal contributions to the core ideas carried out in this study, and obtained funding to support this research.

\section{Additional information}

Supplementary information is available in the online version of the paper. Reprints and permissions information is available online at www.nature.com/reprints. Correspondence and requests for materials should be addressed to S.A.B.

\section{Competing financial interests}

S.A.B. and P.S.C are co-founders of Diagnostic Photonics, Inc., which is licensing intellectual property from the University of Illinois at Urbana-Champaign related to Interferometric Synthetic Aperture Microscopy. S.A.B. received patent royalties from the Massachusetts Institute of Technology for technology related to optical coherence tomography. Other authors declare no competing financial interests. 